\title{
Learning by playing: how to create the perfect learning game for and with optics
}

\section{Tobias Haist, Avinash Burla}

Tobias Haist, Avinash Burla, "Learning by playing: how to create the perfect learning game for and with optics," Proc. SPIE 7783, Optics Education and Outreach, 77830E (30 August 2010); doi: 10.1117/12.860430

SPIE Event: SPIE Optical Engineering + Applications, 2010, San Diego, California, United States 


\title{
Learning by playing - how to create the perfect learning game for and with optics
}

\author{
Tobias Haist, Avinash Burla \\ Institut für Technische Optik, Universität Stuttgart \\ Pfaffenwaldring 9, 70569 Stuttgart, Germany
}

\begin{abstract}
For children, playing and learning is often one thing. They learn while playing and by playing the right games they learn a lot. It is therefore obvious that we should use (among other things) games in order to fascinate children for optics and to teach them the basic laws of optics. In this contribution we will introduce different optical games for children in preschool and elementary school. The majority of commercial learning games on the market do not achieve the ambitious goal of leading to fun and knowledge since very often there are serious design flaws within these games. We introduce ten design rules for learning games that will enable you to create your own successful learning game for a special topic. Exemplary, we will show games based on and for color mixing and polarization.
\end{abstract}

Keywords: Learning, games, optics, polarization, color mixing

\section{INTRODUCTION}

Playing is one of the most important ways how small children learn and for them there is no real difference in learning, playing and working. While not being successfully applicable to all types of learning, games are especially beneficial for certain aspects of the learning process. McFarlane pointed out that "Games promote levels of attention and concentration that teachers, parents and policy makers wished children applied to learning." . ${ }^{1}$ This is easily demonstrated by computer games: A lot of children (and even teenagers) that are not at all motivated in school can sit hour after hour concentrated in front of the computer in order to master a game. Well designed games obviously succeed in motivating the player and motivation is the key element in learning. ${ }^{2}$ Therefore, we should have a closer look to understand how games achieve this strong motivation and how and when we should use games for learning.

In this contribution we will first give a short introduction to game-based learning. Understanding why and how games are interesting for learning is straight forward and, of course, an endless number of "learning games" are available on the market (most of them being quite bad). We will describe a clear recipe of how to design successful learning-games for young (preschool and elementary school) children. We will focus on non-digital games but the results can be easily transferred to all sorts of games. In section 4 and 5 we will turn our attention to examples from optics. Especially, we will explain in detail a game that effectively trains arithmetic skills and subtractive color mixing and we will show two eye-catching games for additive color mixing and polarisation.

\section{GAMES AND LEARNING}

The core ideas behind game-based learning are motivation and flow: A well-known proverb says that "a motivated learner can't be stopped". ${ }^{3}$ Since (good) games are motivating we can conclude that games are good for learning. ${ }^{2}$

Unfortunately, things are not that simple and most of the educational games fail in leading to successful learning. The majority of learning "games" are not really games at all but consist of a succession of small puzzles or exercises that the player has to solve. Real gameplay is not present. Sometimes, these "games" achieve learning success because children like puzzles and thus motivation might be present. However, long lasting motivation or even something like flow (see below) will not be achieved. Even worse, not all children will have the motivation for these typical exercise-based puzzles.

Further author information: Send correspondence to haist@ito.uni-stuttgart.de 
As pointed out by different authors ${ }^{2,4-6}$ a lot of educational games just transfer bad methods of traditional education to a new medium. One important and typical problem is that the player constantly receives negative feedback if he does something wrong. From the psychological standpoint this seems to be beneficial at first. However, taking into consideration that we are talking about games it is counterproductive because it will be difficult if not impossible for the player to reach the state of so called "flow". Flow can be described by a completely focused attention with a clear set of goals and the perception of challenge that is matched to the person's skills. ${ }^{6}$

In this state we are so involved that nothing else matters. Long concentration (even accompanied by some sort of time distortion) can be reached during flow and it has been shown that this leads to very successful learning. ${ }^{6}$ Therefore, we should try to reach flow during learning. ${ }^{7}$ People like to be in the state of flow and the reward of getting into that state is (apart from curiosity) perhaps the most important motivation for playing a game.

Flow is easily destroyed by external stimuli or wrong level of difficulty. For reaching the flow it is most important that the task is challenging, but doable. ${ }^{6}$ Well designed computer-assisted training or computer games that incorporate this (using e.g. "dynamic difficulty adjustment") have strong advantages with respect to this compared to traditional course based learning where there is always a compromise due to the different abilities of the pupils. ${ }^{8}$

Unfortunately, most educational computer-based material neglects this, which is especially a problem with educational computer games where often some kind of hidden teacher is implemented. ${ }^{8}$ Royle describes it perfectly by "just when you were starting to enjoy yourself, the game is interrupted by some thing telling you how you could have done it better." 4 To summarize, if well done, games lead to high motivation, concentration and flow and thus successful learning.

Nevertheless, one should keep in mind that game-based learning is not always the best choice. Other methods like reading text books, attending conventional courses, doing experiments, or solving real tasks in a team might be better suited to teach a certain topic. Especially, experiment-based learning is a very important alternative for optical topics. ${ }^{9,10}$ First of all, game-based learning is not very effective concerning the amount of time that is needed to learn. A highly motivated student with a good text book will learn much more facts in one hour compared to the same student using a learning game because the information density of a game is low compared to a book. Second, games might be so engaging that an educational focus is lost. ${ }^{4}$ Third, playing might be so attractive that other forms of learning are avoided and not trained enough. This is especially dangerous for computer games. Such games are very attractive even for young children, because complete immersion is easily achieved. This should be compared to our most important information source, written text. Here, the same level of immersion is given only after profound reading skills have been developed. The graphical escape into the multimedia world of today's computer software is, therefore, dangerous because it might lead to problems when learning to read.

Game-based learning is, on the other hand, extremely successful for implementing so called "stealth learning". In this case the child will not realize that he or she is learning. This kind of learning is especially important if one wants to drill a special ability (e.g. basic arithmetic or language skills). ${ }^{4}$ Traditional learning methods (e.g. flash cards) in this case soon become ineffective because the endless repetition leads to boredom and demotivation. For such content it is a good idea to hide the continuous repetition within a game (compare this with the game presented in section 4). For the player, learning becomes only a side effect. Playing the game in this case is the main thing.

Also, it should be noted that the best suited learning method depends on the learner itself. As pointed out by Prensky: "Kids like all humans love to learn when it isn't forced upon them." ${ }^{3}$ But this is true only if they are motivated. A pupil being highly motivated for a certain topic might use a learning technique that has a high information density (reading a book). For another child, this technique might be completely inefficient due to the lack of motivation. In such cases, games can act as important tools to enhance motivation.

The challenge of learning games, therefore, is to wrap the content to be learned into a "good" game that the player is highly motivated to play and that keeps the high motivation over a comparatively long time and that sometimes even leads to flow. 


\section{HOW TO CREATE A SUCCESSFUL LEARNING GAME}

As already pointed out it does not make sense to try to teach everything by using games. We should apply game-based learning to material that is prone to demotivation using conventional learning methods and still needs high repetition rates. Therefore, the very first step in designing a learning game is to critically rethink if a game is really the best suited tool for the content to be learned.

Game-based learning is only effective if the game is a "good" game. It is of utmost importance that the game lies in the center of our consideration. Lack of pedagogic quality might lead to imperfect learning but bad game-play completely destroys the basic idea of game-based learning because the learner does not want to play the game. ${ }^{8}$ Motivation and flow will be lost.

So, what makes a good game? Such games typically have some of the following important qualities (see also $^{7,11-13}$ for more and other qualities):

- Challenges with the right level of difficulty (leading to sense of triumph and psychological pleasure)

- Immersion / flow

- Unpredictability of the outcome

- Social interaction with others

- Competition

- Possibility to make own decisions

- Goal orientation

- Enjoyment

This background is important but it is too abstract to really help when designing. To this end we propose to keep the following important ten design rules in mind during the development of a learning game for children:

1. Stealth Learning: Gameplay comes first, didactics and learning is less important. Playing basketball you will gain good fitness but this is not the primary reason why you play it. It is only a side-effect (see also the discussion above).

2. Duration: The game should have a suitable duration, typically 10 to 20 minutes for young children, because concentration at that age typically will fade soon (if not, you still can play a second time).

3. Randomness: The game should not be completely random. A lot of games for children are based on the principle that children should have the same chance to win as grown-ups and that, therefore, the outcome should be random. This is wrong. Not only does the child learn that it does not help to create a strategy and that it does not make sense to learn but even worse, it might lead to the situation that when playing the game the very first time, the child will lose against the grown-up. Typically, young children are very sensitive for such a situation. They anyway fear that they will lose (because grown-ups are more skilful in a lot of aspects) and this fear is hard to overcome. Now, if they indeed lose the first game they are proven right that it does not make sense to play the game because the outcome for them is clear anyway. Logically, they refuse to play a second time. Therefore, it is imperative to make sure when playing against young children to lose at least the first game. It has to be possible to lose at will without the kid noticing that you wanted to lose. This means that the outcome should not be completely random. The right amount of strategic decisions or skills should be incorporated into the game.

Apart from this purely psychological reasons the right amount of strategy and skill is important to increase the motivation for playing (sense of triumph and psychological pleasure) and to train strategic skills. 
4. Decisions/amount of control: To make a game exciting, the player got to have some choice. Too much choice on the other hand is counterproductive and leads to randomness, confusion, or even worse excessive demand. Also, choices are only useful if they have a clear consequence for the course of the game. Single decisions on the other hand might have a strong impact but should not decide about loss or win.

5. Luck: The overall emotion during play should be, of course, positive. So, if there are things like "good" or "bad" fields or cards: Make sure that there are more good ones!

6. Difficulty: The right amount of difficulty is extremely important for successful learning. The game has to be challenging but not too challenging to achieve optimum learning conditions. Within electronic games this can be easily achieved (although it often is not done). With board games it is not that simple and typically it is the task of the grown-up to make sure that the child obtains enough help to finally win (of course still having the impression that he or she won completely on his or her own). In some games one can increase the difficulty very easily (e.g. by incorporating more advanced rules, more difficult tasks). For an example see section 4 .

7. Gimmick: Apart from the gameplay a special gimmick is an important tool to increase the fascination for the game. Typically, this gimmick is without any real use for the gameplay but nevertheless is important.

8. Rules: The rules should be as easy and straight forward as possible (at least the starting rules) so that there is not a large hurdle to overcome and to decrease possible fear of failure for the child. When you explain the game: start as simple as possible and introduce the more advanced rules later. Of course, the rules should be unambiguous and clear.

9. Number of players: These days, it is often beneficial if a game does not need more than two players because often there are not enough players available. One player games with respect to this are of course even better, but social interaction is lost and with non-electronic games it is difficult to adjust the level of difficulty.

10. Gameplay: Last but not least, of course, the game should work. It should be exciting, innovative and it should be unclear (at least until a final stage of escalation) who will win.

For commercial games two additional things should be taken into account that have nothing to do with the game design.

11. Convincing the parent: Parents often buy games for their children because they hope that the games will have a positive learning effect. Therefore, it should be clear (e.g. based on the subtitle of the game) that the game is good for kids. You can even use a topic or a lead character for the game that is somehow associated with good quality (e.g. a character from a famous and highly rated child book).

12. Convincing the kid: Parents also buy games because their kids want them to. Of course, they cannot decide if the gameplay leads to fun. They are attracted by nice gimmicks (e.g. a large plastic crocodile with movable teeth) or attractive topics (e.g. dinosaurs) or characters (e.g. princess Lillifee).

The typical workflow to design a learning (board) game is depicted in Fig. 1. First, and most important is to clearly specify the basic parameters of the game you want to create. One might use the table depicted in Table 1 for this purpose. Second, and of course the most difficult, is the development of the central gameplay. Ideally, you have some innovative ideas that might work. Alternatively, you can combine elements from other games (most of the games are based on that). Anyway, the more different games you personally know, the easier it will be for you to come up with new working combinations or new ideas. Typically, you should have several good ideas that might work.

In the beginning you should concentrate on the rules and the goal of the game. Additional features are introduced later. If you succeeded in having a first satisfying approximation of the game you should critically analyze what is good and bad. This might be not so easy because of lack of enough distance to your creation. 


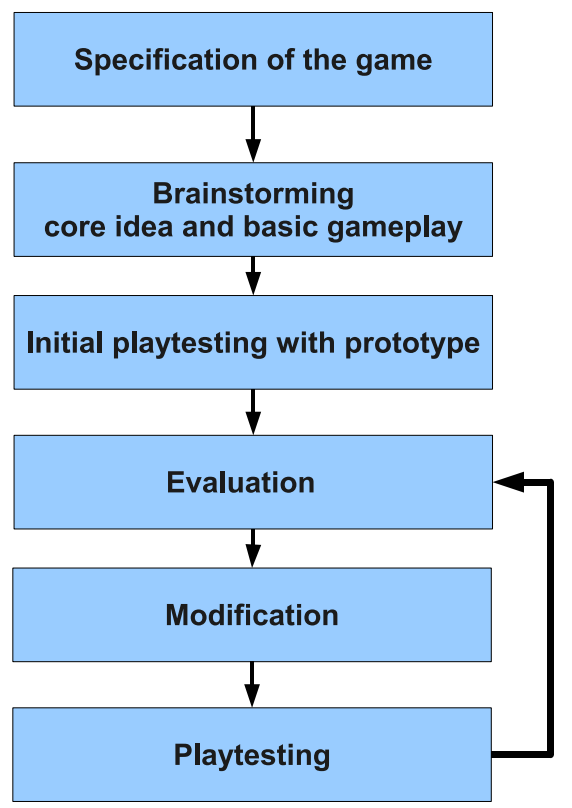

Figure 1. Basic workflow for creating a game.

Therefore, clear analytical investigation of all important aspects is necessary at this stage. It is advantageous to start playtesting as soon as possible because the later you start the more work you will have already invested into the current version and the more you will hesitate to change things.

Apart from the ten rules given above you should now also focus on the emotions that come up while playing. Important emotional payoffs that we wish to achieve are sense of triumph, pride, anger, schadenfreude (a little bit is good!), surprise and of course as an overall result: fun. These emotions have to be balanced against more negative ones like frustration, confusion, boredom, fear. Also at that stage you should be able to describe the core of the game in one or two sentences (if this is not possible, it might be a hint that you still do not completely understand why your game works).

Afterwards, you analyze variations of the basic rules and add additional features. This can be achieved only by a lot of playtesting. It is impossible (even for experienced designers) to foresee what effect a specific change will have on the gameplay or the emotions of the players and, therefore, playtesting is mandatory. Also notice that often rules or features that you thought of being central to the game in practice are not necessary at all. You should remove such unimportant things that detract from the core. Again, the more gaming experience you have the more features you will be able to chose from to repair smaller defects within your design. If you come to the point where you do not see an improvement, it might be fruitful to put the game away for a few weeks in order to get enough distance.

\begin{tabular}{l|l}
\hline Learning Goals & $\begin{array}{l}\text { 1) Subtractive color mixing, } \\
\text { 2) Addition of two and three numbers } \\
\text { 3) Development of basic strategic skills }\end{array}$ \\
\hline Age & 6 to 99 \\
\hline Duration & 10 to 20 minutes \\
\hline Number of players & 2 to 4 \\
\hline Type of Game & Competitive \\
\hline Strategy vs. Chance & $50: 50$ \\
\hline \multicolumn{2}{|c}{ Table 1. Basic specification of the "RGB" game. }
\end{tabular}




\section{RGB}

In the following we describe one successful (according to the thoughts given above) learning game that we call "RGB". It teaches subtractive color mixing and basic arithmetic skills (addition). The complete design of the game (including building a first version) took only three hours and was triggered by the 6-year old daughter (Maya) of one of us. She was fascinated about the colorful transparent dice lying around and wanted to make a game out of them. The basic specification of the game has already been depicted in Table 1.

One could think that it is not really clever trying to teach subtractive color mixing using a game because the most natural way to learn it is to just paint. Without doubt painting is really the best way to learn color mixing but that does not mean that it should be the only way to learn it. It is indeed beneficial to teach the same concept (in this case subtractive color mixing and arithmetic) using different applications and examples because this tightens the learning effect and enriches the understanding. If possible one should always try to envision a concept in different ways to enhance learning.

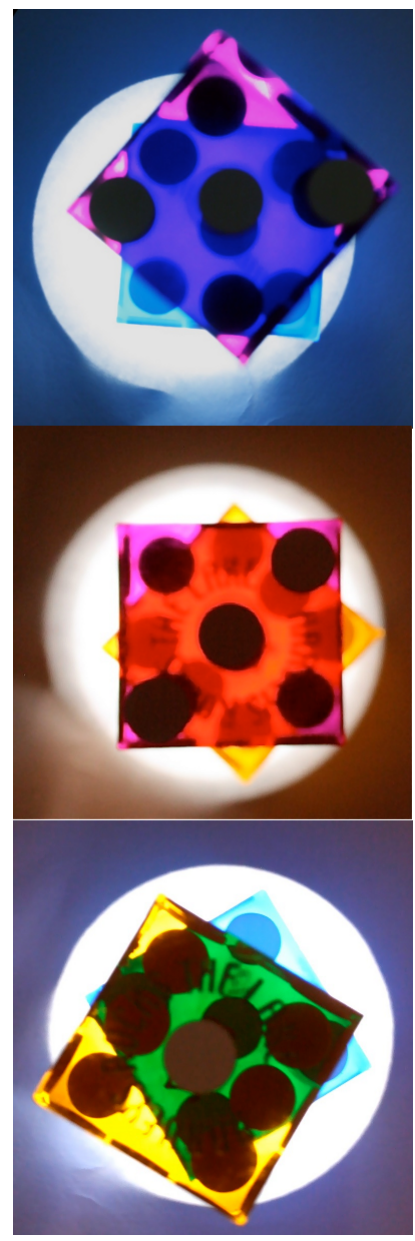

(a) Subtractive color mixing demonstrated by stacked dice.

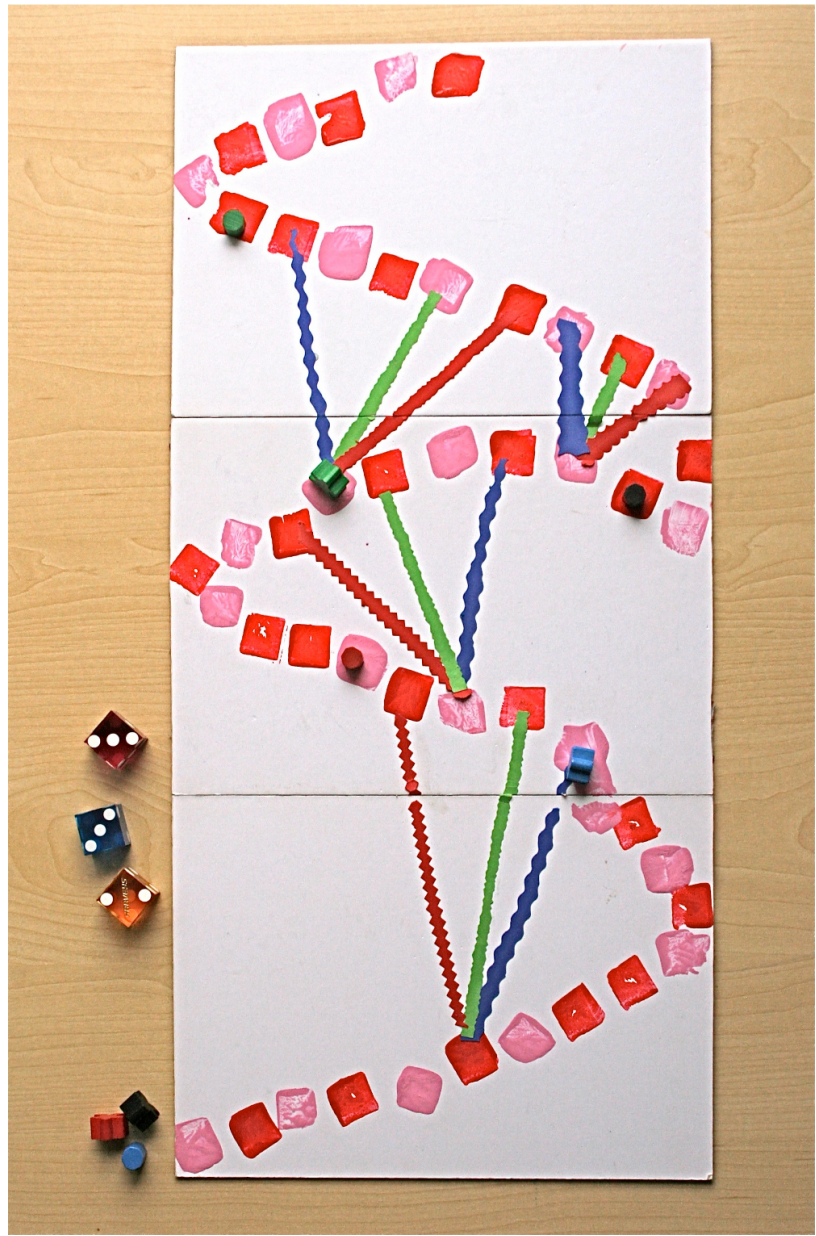

(b) The board.

Figure 2. "RGB": Each player tries to bring all of his four colored (red, green, blue, black) pieces to the final square. The number of squares to move are determined based on subtractive mixing of the dice (see text for details). 
The core of the game consists of three transparent dice (cyan, magenta and yellow). If you stack two dice and illuminate from below you see, of course, the subtractively mixed color (e.g. green if you stack yellow and cyan, see Fig. 2 (a)). This looks very nice and it is fascinating for children to do it (even for grown ups it gives some kind of pleasure to play with this sort of color mixing).

You win the game by bringing all four pieces of yours (red, green, blue, and black) to the goal. If it is your turn you roll all the three dice. You combine the three dice by stacking two or three of them. Then, you move your piece with the corresponding color by the sum of the stacked dice. An example explains this much better: Let us assume that you throw cyan $=5$, magenta $=3$, yellow $=2$. With that you might move red (magenta and yellow) by $3+2=5$ squares, or blue (magenta and blue) by $3+5=8$ squares, or green (cyan and yellow) by $5+2$ $=7$ squares, or black (magenta and yellow and cyan) by $3+2+2=10$ squares.

So you chose one of these combinations and move the correspondingly colored figure of yours.

If you end your move with your piece at a correspondingly colored ladder (e.g. green piece stops at green ladder), the piece will immediately move to the other end of the ladder (up or down).

This is the basic idea and it works surprisingly well. The number of squares has been chosen so that the game has the right duration (10...15 minutes). It can easily be explained and it has the right balance of strategy and luck for a typical six to ten year old child. It is indeed fun to play that game and by doing this a lot of times the arithmetic skills for small numbers (below 20) and the ability to predict color mixing will be indeed improved. At a certain level you can easily extend the arithmetic difficulty by choosing dice with numbers instead of bullets (that way you can avoid that the child counts) or you can use dice with larger numbers (of course in this case you should increase the number of squares). You can also start discussing the law of association for addition and subtractive color mixing after some playing.

Two additional rules (that you only explain after the basic game has been really understood) make the game faster and more interesting: 1) If you role a double (or even a triple) and you moved with the corresponding piece, you are allowed to throw the two dice again and perform another move with that piece. 2) If you end your move on a pink square and one of your opponent's pieces already is occupying this square you can send his piece back to the starting square (beware: children with low tolerance against frustration might not like that at all and you have to introduce this rule later).
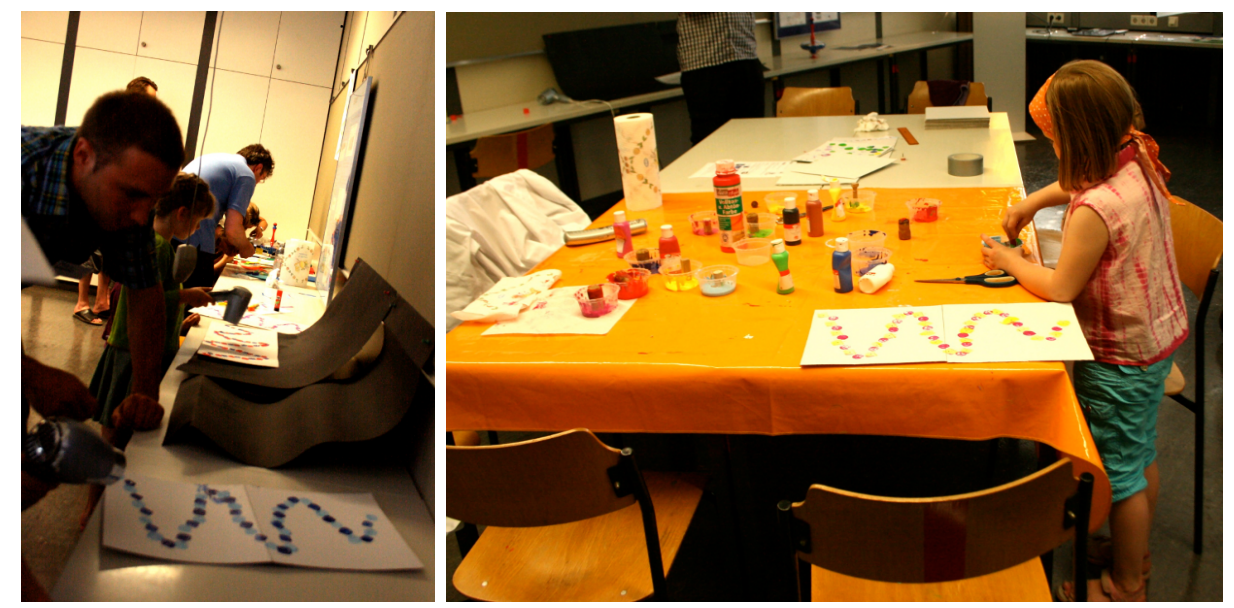

Figure 3. Building the game at the science day at University of Stuttgart

You can easily build a version of that game (see Fig. 2 (b) and Fig. 3). The number of squares and the positions and lengths of the ladders have been chosen carefully and are important. For example, the first ladder starts at square number six because the probability is high that you will get a six and, therefore, the game directly starts with a positive emotion ("I am so lucky!"). You might even throw a double while getting the six. 


\section{GAMES FOR FASCINATING CHILDREN}

Apart from a classical learning game as presented in the last section other sorts of games are of more indirect use. If you just want to create positive emotions for a certain topic (in our case optics) it is best to start with something that is very short and fascinating right from the beginning. No explanations at all should ideally be necessary to arouse interest.

Colored shadows are perfect with respect to this. The setup is simple. You need three slide projectors (or data projectors) and which are arranged close to each other (separation of projection lenses: some tens of centimeters). Red, green and blue filters are used to project large red, green and blue rectangular areas onto a white screen located at a certain distance. Of course, aligning the projectors so that the corresponding projections will fall onto each other will result in a white area on the screen.

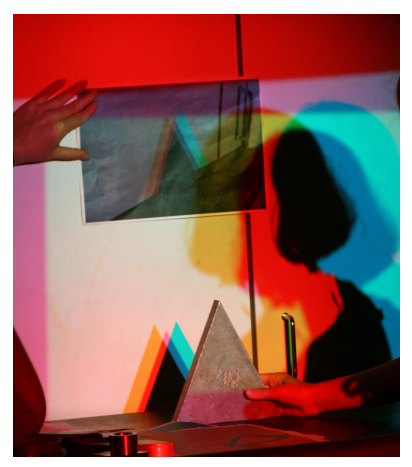

(a) triangle

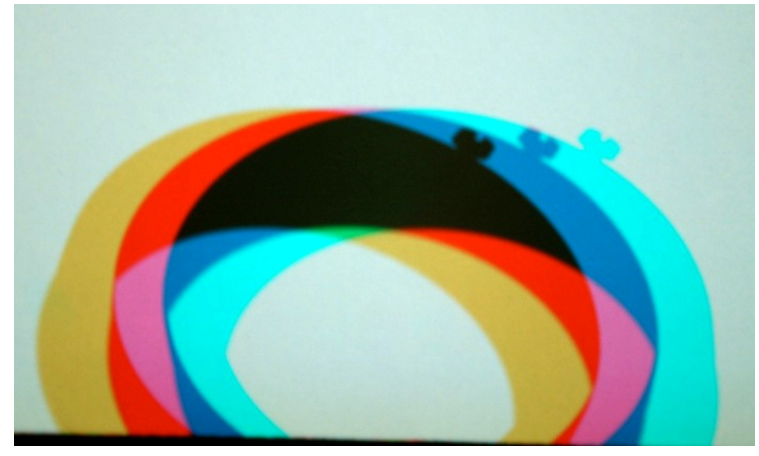

(b) ring

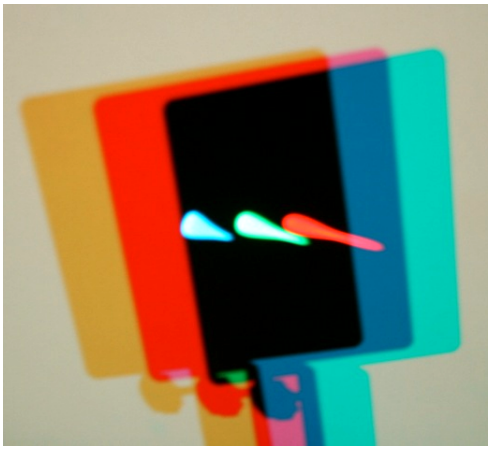

(c) strongly tilted lens

Figure 4. Using three projectors (red, green, blue) one can achieve beautifully colored shadows. The player has to align objects in such a way that the color shadows resemble a certain pattern (shown on the photo to the player).

Now, if you put something (e.g. your body) between the projectors and the screen, you will see a very nice colorful shadow on the screen because the individual shadows due to the three projectors will be at different positions and, therefore, the additive color mixing becomes quite complex. If done correctly, the large colorful and moving shadows look really fascinating (see Fig. 4 and by Costa et al. ${ }^{14}$ ).

You can use the following game to get the children more involved: You hand them a photograph of a prerecorded complicated shadow. Now it is the task of the child to create the same shadow using the objects given to him. This can soon get very complicated.

Of course, you can now easily explain (if you and the child want that) some basic things about colormixing (e.g. switch one or two of the projectors off and start explaining). Or you just enjoy the fact that the child is fascinated by optics.

For pupils and students, polarization is a rather difficult topic lacking the vividness of most other optical phenomena. Direct observation of polarization is not really possible (neglecting the not very spectacular Haidinger brush phenomenon) and, therefore, motivation to understand polarization is often not very high.

It is intimating to try to raise motivation by surprising demonstrations. Apart from the well known experiments (two or three polarizers in series, strain measurement by birefringence, polarizers in photography, just to name a few) we tried raising interest by using polarization-based games.

Fig. 5 shows a birefringence-based puzzle. Here, we use the lids of plastic boxes and some polarising films. The polarizing film is pasted onto the lids. The playing area is just a glass plate illuminated with polarized light (the easiest way of implementation is to use an LCD screen).

The birefringent plastic is illuminated with polarized light. After passage through plastic and the second polarizer (the analyzer) this leads to a colorful pattern because of the birefringence of the plastics. Of course, the pattern depends on the strain and the thickness of the plastic between the polarizers. 


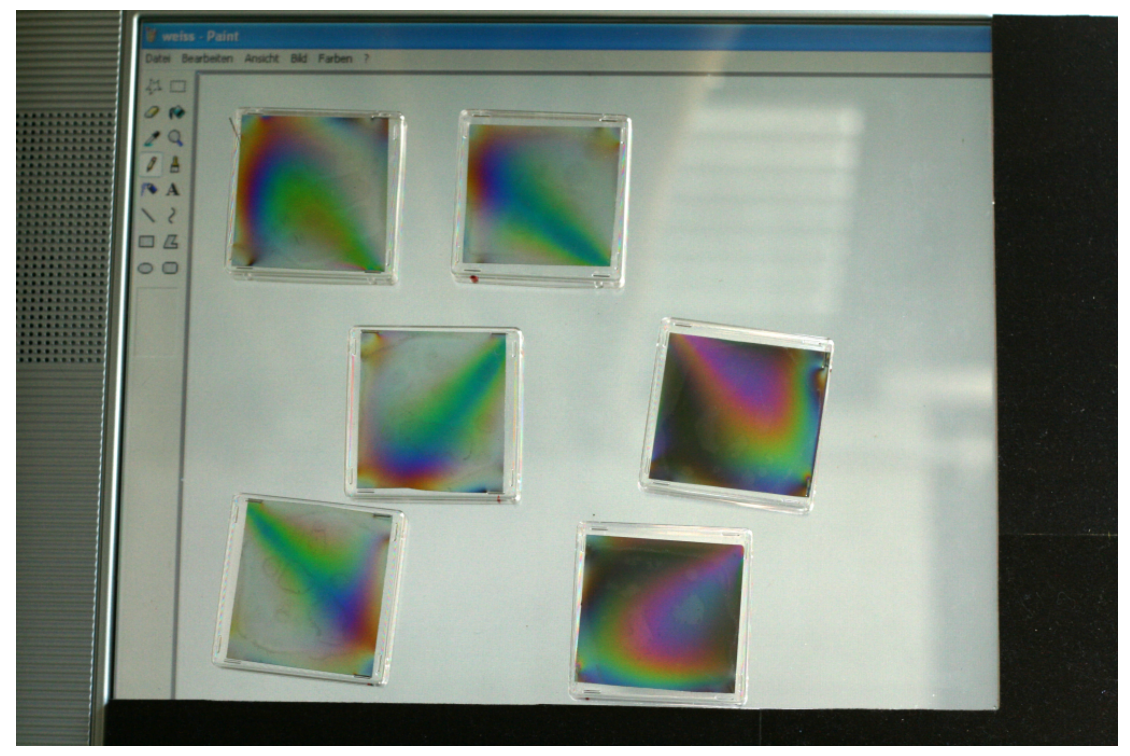

(a) Original setup

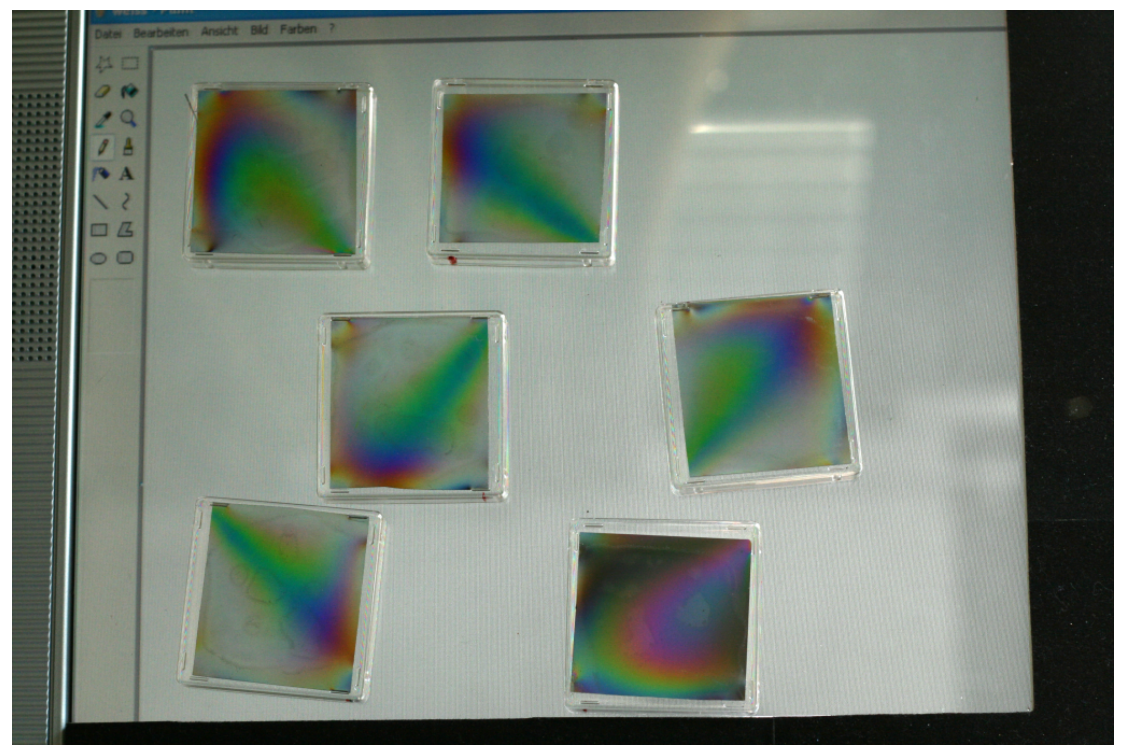

(b) Same setup with one piece rotated

Figure 5. Polarization Puzzle: The pieces have to be positioned and orientated so that a given pattern becomes visible. The pieces change their color if they are rotated because of the birefringence of the plastic.

The aim of the game is to align 9 (or 16) pieces correctly so that they resemble the "solution pattern" which has been printed out before and is given to the player. This, at first, sounds simple because it is a puzzle with only 9 pieces. But the pieces change as you rotate them. This is the really fascinating thing. Also, the game can be easily explained (even to small children) and it can be played alone (with only one visitor on a science event). Therefore, it is indeed very much suited for occasions and events like trade-shows or science days.

The colorful birefringence phenomenon is unfortunately quite complicated. You have to know at least some 
details about waves and interference to understand how the colors arise. Therefore, it might be only used for students studying optics. For younger children it is way too complicated. Nevertheless, it fascinates them.

\section{CONCLUSIONS}

The "polarization-puzzle" and the color shadow game are visually interesting and they can be used for tradeshows, other special events, or science parks. It might help fascinating children for optics and once they are fascinated they might even be interested enough to let you explain some basic things. Designing such games is quite simple. You start with a fascinating optical effect and do not care much about the gameplay.

Such games typically are played only once or twice. If you want to use games for real learning you need a different approach. Games are very effective with respect to drilling special skills where conventional learning soon becomes boring. For such applications it is of utmost importance that the gameplay is good enough to keep the motivation high. Based on careful planning and the design rules explained in section 3 you can create such games.

\section{REFERENCES}

[1] J. Kirriemuir and A. McFarlane, "Literature Review in Games and Learning," FutureLab Series, FutureLab Report 8, pp. 1-39, 2004.

[2] G. Denis and P. Jouvelot, "Motivation-driven educational game design," Proceedings of the 2005 ACM SIGCHI International Conference on Advances in computer entertainment technology - ACE '05, pp. 462465, 2005.

[3] M. Prensky, "Digital game-based learning," Computers in Entertainment 1(1), p. 21, 2003.

[4] K. Royle and R. Clarke, "Making the case for computer games as a learning environment.," Report University of Wolverhampton, School of Education, avail. online http:www.desq.co.ukdoomedpdf Making_the_case.pdf , pp. 1-14, 2003.

[5] F. L. Greitzer, O. A. Kuchar, and K. Huston, "Cognitive science implications for enhancing training effectiveness in a serious gaming context," Journal on Educational Resources in Computing 7(3), pp. 2-es, 2007.

[6] K. Kiili, "Digital game-based learning: Towards an experiential gaming model," The Internet and Higher Education 8(1), pp. 13-24, 2005.

[7] F. Fu, R. Su, and S. Yu, "EGameFlow: A scale to measure learners enjoyment of e-learning games," Computers \& Education 52(1), pp. 101-112, 2009.

[8] N. Peirce, O. Conlan, and V. Wade, "Adaptive Educational Games: Providing Non-invasive Personalised Learning Experiences," in DIGITEL '08: Proceedings of the 2008 Second IEEE International Conference on Digital Game and Intelligent Toy Enhanced Learning, pp. 28-35, IEEE Computer Society, 2008.

[9] N. Magnani and J. Donnelly, "Innovative Methods to Teach Optics in the Grade 5 Classroom," Proc. SPIE ETOP 2007 (Conf. on Education and Training in Optics and Photonics) avail. online http://spie.org/etop/2007/etop07k12V.pdf , 2008.

[10] D. R. Sokoloff, "Active Learning of Introductory Optics: Interactive Lecture Demonstrations and Optics Magic Tricks," Proc. SPIE ETOP 2007 (Conf. on Education and Training in Optics and Photonics) avail. online http://spie.org/etop/2007/etop07methodsIII.pdf , 2008.

[11] T. Fullerton, C. Swain, and S. Hoffman, Game Design Workshop: Designing, Prototyping, and Playtesting Games, CMP Books, 2004.

[12] K. Zimmerman, E., Salem, Rules of Play: Game Design Fundamentals, MIT Press, 2004.

[13] B. Tinsman, The Game Inventor's Guidebook, Morgan James Publishing, 2008.

[14] M. F. M. Costa, M. Lira, and S. Franco, "Kindergarten optics," Proc. SPIE ETOP 2009 (Conf. on Education and Training in Optics and Photonics) avail. online http://spie.org/etop/2009/etop2009_3b.8.15.pdf , 2009. 